\title{
Numerical solution of filtration in porous rock
}

\author{
Galina Safina* \\ Moscow State University of Civil Engineering, Yaroslavskoe shosse, 26, Moscow, 129337, Russia
}

\begin{abstract}
The filtration problem is one of the most relevant in the design of retaining hydraulic structures, water supply channels, drainage systems, in the drainage of the soil foundation, etc. Construction of transport tunnels and underground structures requires careful study of the soil properties and special work to prevent dangerous geological processes. The model of particle transport in the porous rock, which is based on the mechanicalgeometric interaction of particles with a porous medium, is considered in the paper. The suspension particles pass freely through large pores and get stuck in small pores. The deposit concentration increases, the porosity and the permissible flow of particles through large pores changes. The model of one-dimensional filtration of a monodisperse suspension in a porous medium with variable porosity and fractional flow through accessible pores is determined by the quasi-linear equation of mass balance of suspended and retained particles and the kinetic equation of deposit growth. This complex system of differential equations has no explicit analytical solution. An equivalent differential equation is used in the paper. The solution of this equation by the characteristics method yields a system of integral equations. Integration of the resulting equations leads to a cumbersome system of transcendental equations, which has no explicit solution. The system is solved numerically at the nodes of a rectangular grid. All calculations are performed for non-linear filtration coefficients obtained experimentally. It is shown that the solution of the transcendental system of equations and the numerical solution of the original hyperbolic system of partial differential equations by the finite difference method are very close. The obtained solution can be used to analyze the results of laboratory research and to optimize the grout composition pumped into the porous soil.
\end{abstract}

\section{Introduction}

The study of water filtration in soils and various porous materials is of great practical interest in solving a variety of engineering problems in the field of hydraulic engineering, construction of water supply and wastewater facilities. Calculations of filtration problems are used in the design of retaining hydraulic structures (earthen dams, lintels, dams), water supply channels, drainage systems, when draining the soil foundation, etc. In the construction of underground structures, cementation of soils is the most common method of

\footnotetext{
*Corresponding author: minkinag@ mail.ru
} 
strengthening porous rock [1]. Therefore, research and development of improved solutions for the soil strengthening is one of the most pressing problems in the field of construction.

Solid particles are carried by the fluid flow through the sinuous channels of a porous medium, some particles are locked in the pores and are retained on the porous frame, which leads to a change in its structure, reducing its porosity and accessible fractional flow. [2]. There are various mechanisms of particle capture by porous rocks: size exclusion in thin pores, bridging, gravity segregation, and diffusion [3], [4].

A mathematical model of particle motion in a filter based on size-exclusion mexanism of particle retention in a porous medium is considered in [5], [6] . The suspension particles freely pass through large pores and lock pores that are smaller than the particle size. The considered model takes into account the change in porosity and accessible particle flow through large pores with increasing deposit concentration. At the initial time the porous medium does not contain suspended and retained particles. The suspension of constant concentration is injected at the filter inlet. It gradually fills the porous medium. The concentration front of suspended and retained particles moves with a constant velocity from the inlet to the outlet. Before the front, the porous medium is empty and the concentrations of suspended and retained particles are zero. Behind the front, particles are filtered, the concentrations of suspended and retained particles are positive.

There are various filtration models of monodisperse and polydisperse suspensions [7], [8]. In a number of important special cases, it is possible to obtain-an exact or asymptotic solution of the problem [9], [10]. In the general case the model have no explicit analitic solution and numerical methods have to be used. The filtration problem is reduced to a system of transcendental equations. In this paper, the system solution was found using the Matlab application package. The solution of the transcendental system is compared with the numerical solutions of the original hyperbolic system and the equivalent partial differential equation, obtained using difference schemes with second-order approximation.

\section{A mathematical model}

Quasilinear hyperbolic system of first order equations

$$
\begin{gathered}
\frac{\partial(g(S) C+S)}{\partial t}+\frac{\partial(f(S) C)}{\partial x}=0, \\
\frac{\partial S}{\partial t}=\Lambda(S) C .
\end{gathered}
$$

determines a one-dimensional filtration model of a monodisperse suspension in a porous medium with variable porosity $g(S)$ and accessible fractional flow $f(S)$. Here $C(x, t)$ and $S(x, t)$ are concentrations of suspended and retained particles of the porous medium, $\Lambda(S)$ is the filtration coefficient.

The mathematical model (1), (2) is considered in the domain $\Omega=\{0 \leq x \leq 1, t \geq 0\}$, while the suspension with a constant concentration of suspended particles is injected at the inlet of the porous medium $x=0$

$$
\left.C(x, t)\right|_{x=0}=1,
$$

at the initial time $t=0$ the concentrations of suspended and retained particles of the porous medium are zero

$$
\left.C(x, t)\right|_{t=0}=0,\left.\quad S(x, t)\right|_{t=0}=0 .
$$


The concentration front of suspended and retained particles, determined by a linear equation $t=\frac{g(0)}{f(0)} x$, moves in a porous medium with a constant velocity $v=\frac{f(0)}{g(0)}$ and divides the domain $\Omega$ into two subdomains

$$
\Omega_{0}=\left\{(x, t): 0 \leq x \leq 1,0 \leq t<\frac{g(0)}{f(0)} x\right\} ; \Omega_{1}=\left\{(x, t): 0 \leq x \leq 1, t \geq \frac{g(0)}{f(0)} x\right\} .
$$

In the domain $\Omega_{0}$ the solutions $C(x, t)$ and $S(x, t)$ are zero, in the domain $\Omega_{1}$ both solutions are positive. At the concentration front the solution $C(x, t)$ has a discontinuity of the first kind, the solution $S(x, t)$ is continuous and has discontinuous derivatives.

In [11] it is shown that from the system of equations (1)-(4) it is possible to obtain the equation

$$
g(S) \frac{\partial S}{\partial t}+f(S) \frac{\partial S}{\partial x}+S \Lambda(S)=0
$$

with initial condition

$$
\left.S(x, t)\right|_{x=0}=S_{0}(t),
$$

where the initial function is determined implicitly by the relation

$$
\int_{0}^{S_{0}(t)} \frac{d s}{\Lambda(s)}=t
$$

Let us rewrite equation (5) as

$$
\frac{g(S)}{\Lambda(S)} \frac{\partial S}{\partial t}+\frac{f(S)}{\Lambda(S)} \frac{\partial S}{\partial x}+S=0
$$

To solve the last equation in the domain $\Omega_{1}$, we use the characteristics method. Consider the characteristic curves with the beginning on the axis $O t$. Let us introduce new characteristic variables such that $t_{0}$ is the starting point of the characteristic on the axis $O t$ and $\tau$ is the parameter along the characteristic. Then the characteristics are determined by the equations

$$
\frac{d t}{d \tau}=\frac{g(S)}{\Lambda(S)},\left.t\right|_{\tau=0}=t_{0}, \quad \frac{d x}{d \tau}=\frac{f(S)}{\Lambda(S)},\left.x\right|_{\tau=0}=0,
$$

equation (8) takes the form

$$
\frac{d S}{d \tau}+S=0
$$

and condition (6) is written in the form:

$$
\left.S\right|_{\tau=0}=S_{0}\left(t_{0}\right)
$$

The solution of the problem (10), (11) has the form

$$
S\left(t_{0}, \tau\right)=S_{0}\left(t_{0}\right) e^{-\tau} \text {. }
$$

To obtaine the solution to problem (5), (6), it is necessary to express characteristic variables $\tau, t_{0}$ in formula (12) in terms of Cartesian variables $x$, $t$. Substituting expression (12) into equations (9) and integration over the variable $\tau$ with respect to initial conditions yields:

$$
t=\int_{0}^{\tau} \frac{g\left(S_{0}\left(t_{0}\right) e^{-\tau}\right)}{\Lambda\left(S_{0}\left(t_{0}\right) e^{-\tau}\right)} d \tau+t_{0} ; \quad x=\int_{0}^{\tau} \frac{f\left(S_{0}\left(t_{0}\right) e^{-\tau}\right)}{\Lambda\left(S_{0}\left(t_{0}\right) e^{-\tau}\right)} d \tau
$$

The variable replacement in integrals 


$$
S=S_{0}\left(t_{0}\right) e^{-\tau} \Rightarrow d \tau=-\frac{d S}{S}
$$

allows to simplify expressions:

$$
\begin{gathered}
t=-\int_{S_{0}\left(t_{0}\right)}^{S} \frac{g(S)}{S \Lambda(S)} d S+t_{0} ; \\
x=-\int_{S_{0}\left(t_{0}\right)}^{S} \frac{f(S)}{S \Lambda(S)} d S .
\end{gathered}
$$

At $\tau=0$ we have $t=t_{0}$ and formula (7) takes the form

Let us substitute (15) into (13)

$$
\int_{0}^{s_{0}\left(t_{0}\right)} \frac{d s}{\Lambda(s)}=t_{0}
$$

$$
t=-\int_{S_{0}\left(t_{0}\right)}^{S} \frac{g(S)}{S \Lambda(S)} d S+\int_{0}^{S_{0}\left(t_{0}\right)} \frac{d s}{\Lambda(s)}
$$

So, the system of equations (5), (6) is reduced to a system of integral equations

$$
\begin{gathered}
\int_{0}^{S_{0}} \frac{d S}{\Lambda(S)}-\int_{S_{0}}^{S} \frac{g(S)}{S \Lambda(S)} d S=t \\
-\int_{S_{0}}^{S} \frac{f(S)}{S \Lambda(S)} d S=x .
\end{gathered}
$$

For known solution $S(x, t)$ the solution $C(x, t)$ can be determined from the relation between the solutions obtained in [12].

\section{Numerical methods}

The system of equations (16), (17) is solved under the assumption that functions $g(S), f(S), \Lambda(S)$ are the cubic polynomials:

$$
\begin{aligned}
& g(S)=g_{0}+g_{1} S+g_{2} S^{2}+g_{3} S^{3} ; \\
& f(S)=f_{0}+f_{1} S+f_{2} S^{2}+f_{3} S^{3} ; \\
& \Lambda(S)=\lambda_{0}+\lambda_{1} S+\lambda_{2} S^{2}+\lambda_{3} S^{3},
\end{aligned}
$$

moreover, the filtration coefficient is zero at a certain positive value $S$, otherwise the concentration of retained particles increases indefinitely and is infinite in the limit.

Then the cubic polynomial $\Lambda(S)$ can be represented as

$$
\Lambda(S)=(S-\tilde{S})\left(\lambda_{3} S^{2}+\lambda_{1}^{\prime} S+\lambda_{0}^{\prime}\right)
$$

where $\tilde{S}$ is the root of equation $\Lambda(S)=0$.

Each integrand of equations (16), (17) can be represented as a sum of elementary fractions that can be integrated easily:

$$
\int_{0}^{S_{0}}\left(\frac{a}{(S-\tilde{S})}+\frac{b S+c}{\lambda_{3} S^{2}+\lambda_{1}^{\prime} S+\lambda_{0}^{\prime}}\right) d S-\int_{S_{0}}^{S}\left(\frac{d}{S}+\frac{e}{(S-\tilde{S})}+\frac{h S+k}{\lambda_{3} S^{2}+\lambda_{1}^{\prime} S+\lambda_{0}^{\prime}}\right) d S=t
$$




$$
\int_{S_{0}}^{S}\left(\frac{l}{S}+\frac{m}{(S-\tilde{S})}+\frac{n S+p}{\lambda_{3} S^{2}+\lambda_{1}^{\prime} S+\lambda_{0}^{\prime}}\right) d S=-x .
$$

The integration of equations (18), (19) yields a cumbersome system of transcendental equations. The solution of this system is not expressed in elementary functions. We will solve this system numerically. Let us introduce a rectangular grid in a region $\Omega$ with a step $h_{x}$ along the coordinate axis $x$ and $h_{t}$ along the axis $t$ :

$$
x_{i}=i h_{x}, t_{j}=j h_{t} ; i=0,1,2, \ldots, n ; j=0,1,2, \ldots, m,
$$

the step $h_{x}=1 / n$, where $n$ is the number of partitions $[0,1]$, and the step $h_{t}$ is selected from the Courant convergence condition [13]

$$
h_{t} \leq \alpha h_{x}, \quad \alpha=\min _{S \geq 0}(g(S) / f(S)) .
$$

Then, at each node of the domain $\Omega_{1}$ it is possible to solve the system of equations (18), (19), substituting the current values $t_{j}$ and $x_{i}$ into the right-hand sides of the equations. Values of $C(x, t)$ are calculated in the same nodes.

To test the obtained solution the problem (1)-(4) was numerically solved by the method of finite differences, similarly to [14]-[16]. The integrals from the left side of equation (1) over a rectangular grid cell were approximated by the formulas of trapezoids and rectangles, which have a second order of accuracy. The replacement of the partial derivative in equation (2) with second-order difference relations at intermediate nodes and at the boundary with $t_{j}=m h_{t}$

$$
\frac{\partial S}{\partial t} \approx \frac{S_{i, j+1}-S_{i, j-1}}{2 h_{t}}, \quad \frac{\partial S}{\partial t} \approx \frac{S_{i, j-1}-4 S_{i, j}+3 S_{i, j+1}}{2 h_{t}}
$$

also gives the second order of accuracy of a difference schem approximation.

\section{Calculation results}

The calculation of filtration problems (1)-(4), (5)-(6) and (20)-(21) are carry out for the coefficients

$$
\begin{gathered}
g(S)=0.7635+2.44 \cdot 10^{-15} S+3.2 \cdot 10^{-14} S^{2}+3.6 \cdot 10^{-13} S^{3} \\
f(S)=0.9075+2.315 \cdot 10^{-4} S+2.27 \cdot 10^{-8} S^{2}-3.42 \cdot 10^{-8} S^{3} \\
\Lambda(S)=1.551-3.467 \cdot 10^{-3} S-1.16 \cdot 10^{-6} S^{2}-1.16 \cdot 10^{-7} S^{3}
\end{gathered}
$$

obtained in laboratory experiments for particles with a radius $r=3.168 \mu \mathrm{m}$ [11].

Numerical modeling of problems (1)-(4) and (5)-(6) is performed by the finite difference method with steps $h_{x}=0.01, h_{t}=\frac{g(0)}{f(0)} h_{x}$. The solution of the transcendental system of equations (18)-(19) is obtained using Matlab software package.

In Figures 1, 2 the graphs of suspended and retained particles concentrations of numerical solutions at the outlet of the porous medium $x=1$ are shown. The solid line corresponds to the solution of the problem (18)-(19), the dotted line - to the solution of the problem (5)-(6) by the trapezoid method, the dash-and-dotted line - to the solution of the 
problem (1)-(4) using the rectangle formula. In Figure 1 (a) and Figure 2 (a) the graphs are presented in the general form for $0 \leq t \leq 800$, in Figure 1 (b), (c) and Figure 2 (b), (c) the graphs are shown in an enlarged form for $100 \leq t \leq 100.01$ and $500 \leq t \leq 500.01$, respectively.

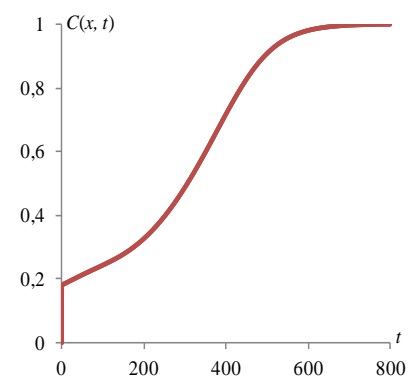

a)

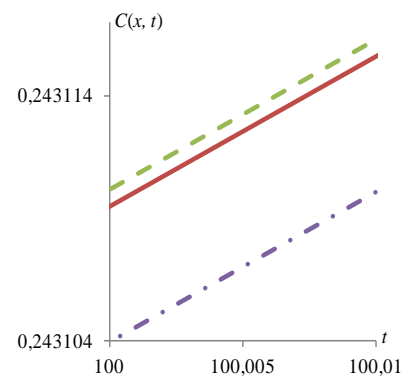

b)

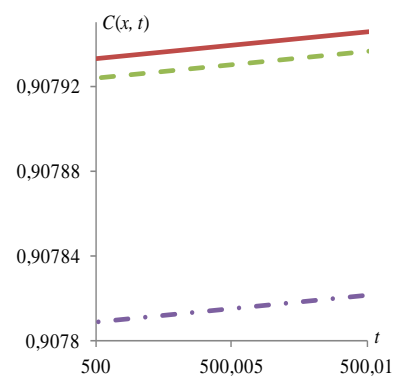

c)

Fig. 1. The suspended particle concentration $\left.C(x, t)\right|_{x=1}$ a) general view; b) enlarged view for $100 \leq t \leq 100.01$; c) enlarged view for $500 \leq t \leq 500.01$.

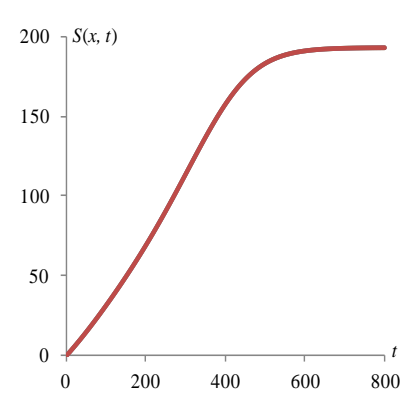

a)

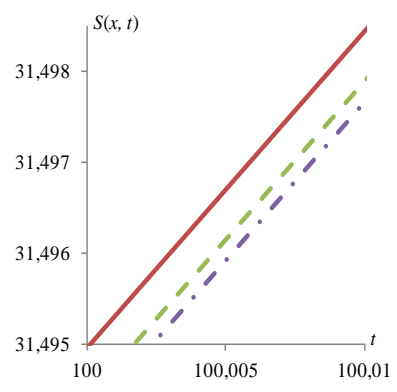

b)

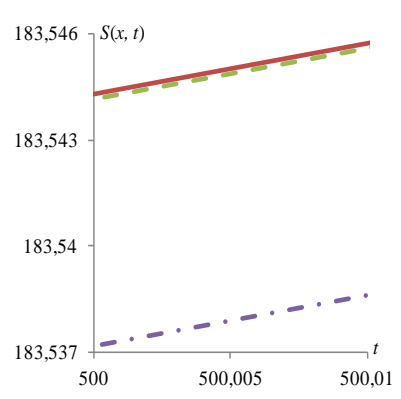

c)

Fig. 2. The retained particle concentration $\left.S(x, t)\right|_{x=1}$ a) general view; b) enlarged view for $100 \leq t \leq 100.01$; c) enlarged view for $500 \leq t \leq 500.01$.

Figures 1, 2 show that the numerical solutions are very close to the solution of the transcendental system (18)-(19), while the trapezoid method is more accurate in comparison with the rectangle method.

\section{Discussion}

The one-dimensional filtration problem of monodisperse suspension in a porous medium with variable porosity and accessible fractional flow is considered in the paper. A mathematical model based on size-exclusion particles capture is a system of partial differential equations of the first order. Continuing the works [17], [18], this article presents a numerical solution of the problem.

The standard approach for the numerical solution of the filtration problem is to solve the original nonlinear system (1)-(4) using the finite difference method [19], [20]. In contrast to the generally accepted method, in this paper the filtration problem is reduced to a system of transcendental equations that are solved numerically at the nodes of a rectangular grid. This 
method increases the accuracy and reduces the time of calculations. The results of the calculations are close to the standard numerical solution of the original problem.

The obtained solution of filtration problem allows to analyze data from laboratory experiments and optimize the composition of grout for the porous rock.

\section{Conclusions}

The problem (1)-(4) of one-dimensional filtration of a monodisperse suspension in a porous medium with variable porosity and accessible fractional flow does not have an explicit analytical solution. The paper considers the transition from system (1)-(2) to the equivalent equation (5) and the solution of this equation by the characteristics method. The obtained transcendental system of equations (18)-(19) is solved numerically at each node of the rectangular grid in the domain $\Omega_{1}$.

The solution of the filtration problem (18)-(19) is compared with the numerical solutions of problems (1)-(2) and (9), obtained by the finite difference method with the second order approximation by integrating over a rectangular grid cell $\Omega_{i, j}=\left\{x_{i-1} \leq x \leq x_{i}, t_{j-1} \leq t \leq t_{j}\right\} \quad$ using the trapezoidal and rectangle formulas. Calculations show that numerical solutions are close. The maximum relative errors of the solutions obtained by the trapezoid and rectangle method in relation to the solution of the transcendental system on the entire time interval $0 \leq t \leq 800$ are $0.004 \%$ and $0.95 \%$, respectively.

\section{References}

1. S. Li, R. Liu, Q. Zhang Q, X. Zhang X, Protection against water or mud inrush in tunnels by grouting: a review, J. of Rock Mechanics and Geotechnical Engineering, 8 (2016) pp. 753-766.

2. T. Russell, L. Chequer, S. Borazjani, Z. You, A. Zeinijahromi, P. Bedrikovetsky, Formation Damage by Fines Migration: Mathematical and Laboratory Modelling, Field Cases, Formation Damage During Improved Oil Recovery: Chapter 3 (2018) pp. 69-175.

3. F. Civan, Reservoir formation damage (Huston: Gulf Professional Publishing, 2015).

4. Y. Osipov, N. Kotov, Asymptotic model of size-exclusion grouting, Materials Science and Engineering, 365 (2018) 042006.

5. L.I. Kuzmina, Y.V. Osipov, Y.G. Zheglova, Analytical model for deep bed filtration with multiple mechanisms of particle capture, International Journal of Non-Linear Mechanic, 105 (2018) pp. 242-248.

6. L. Kuzmina, Y. Osipov, Deep bed filtration with multiple pore-blocking mechanisms, MATEC Web of Conferences, 196 (2018) 04003.

7. L. Kuzmina, Y. Osipov, Filtration model of the unsteady suspension flow in a porous medium, MATEC Web of Conferences, 117 (2017) 00097.

8. Y. Osipov, Calculation of the filtration of polydisperse suspension with a small rate MATEC Web of Conferences, 117 (2017) 00131.

9. S. Borazjani, P. Bedrikovetsky, Exact solutions for two-phase colloidal-suspension transport in porous media, Applied Mathematical Modelling, 44 (2017) pp. 296-320.

10. L.I. Kuzmina, Yu.V. Osipov, Asymptotic model of filtration in almost stationary mode, International Journal for Computational Civil and Structural Engineering, 12(1) (2016) pp. 158-163. 
11. P. Bedrikovetsky, Z. You, A. Badalyan, Y. Osipov, L.Kuzmina, Analytical model for straining-dominant large-retention depth filtration, Chemical Engineering Journal, 330 (2017) pp. 1148-1159.

12. E.A. Vyazmina, P.G. Bedrikovetskii, A.D. Polyanin, New classes of exact solutions to nonlinear sets of equations in the theory of filtration and convective mass transfer, Theoretical Foundations of Chemical Engineering, 41(5) (2007) pp. 556-564.

13. E. F. Toro, Riemann solvers and numerical methods for fluid dynamics (Dordrecht: Springer, 2009)

14. Y. P. Galaguz, G.L. Safina, Modeling of fine migration in a porous medium, MATEC Web of Conferences, 86 (2016) 03003.

15. Y. P. Galaguz, G.L. Safina, Calculation of the filtration in a heterogeneous porous medium, MATEC Web of Conferences, 117 (2017) 00052.

16. Y. P. Galaguz, G.L. Safina, Modeling of filtration of 2-types particles suspension in a porous medium, MATEC Web of Conferences, 117 (2017) 00053.

17. Yu. Osipov, G. Safina, N. Vetoshkin, Calculation of the filtration at the porous medium outlet, Matec Web of Conferences, 196 (2018) 04021.

18. Yu. Osipov, G. Safina, Yu. Galaguz, Calculation of the filtration problem by finite differences methods, MATEC Web of Conferences, 251 (2018) 04021.

19. L.I. Kuzmina, Yu.V. Osipov, Yu.P. Galaguz, Numerical and asymptotic modelling of a filtration problem with the initial deposit, International Journal for Computational Civil and Structural Engineering, 3 (2017) pp. 70-76.

20. L.I. Kuzmina, Yu.V. Osipov, Yu.G. Zheglova, Modeling uniform asymptotics of the filtration problem in a porous medium, Materials Science and Engineering, 456 (2018) 012051. 